\title{
PRACTICAL SYNTHESIS METHOD FOR HEAT EXCHANGER NETWORK
}

\author{
MASAAKI MURAKI AND TOYOHIKO HAYAKAWA \\ Industrial Engineering Department, \\ Tokyo Institute of Technology, Tokyo 152
}

\begin{abstract}
Synthesis of an optimal (minimum-cost) heat exchanger network is very important from the viewpoint of energy saving and cost reduction, but it is difficult to solve these synthesis problems because there exist an extremely large number of possible stream combinations to be considered. A practical method composing three stages [pre-analysis, generation of initial networks, and evolution] was developed to synthesize an optimal network by hand calculation. The problem table is adopted to determine the minimum utility requirement precisely, and to search necessary conditions of maximum heat recovery in the pre-analysis stage. A simple algorithm of initial network generation is developed to generate nearly optimal networks considering maximum heat recovery and optimal locations of heaters and coolers. Initial networks are improved by simple evolutionary rules based on stream-splitting to increase the heat duties of units and to vary the exchanger sizes in the evolution stage. The effectiveness of this method is demonstrated by solving the synthesis problems reported in previous works.
\end{abstract}

\section{Introduction}

Heat exchanger networks are widely used to reduce energy consumption in the petroleum and petrochemical industries, and the utility and capital cost to accomplish the required heat exchange can often account for the major portion of the plant cost. With the recent energy cost increase and quantitative limits on supply of energy, it is very important to solve the problem of synthesizing an optimal (minimum-cost) network of exhangers, heaters, and/or coolers in view of energy saving and cost reduction.

Many different methods have been proposed to synthesize an optimal network, and recent reviews can be found in Nishida et al. ${ }^{6)}$ and Hlavacek ${ }^{3)}$. The difficulties of synthesizing an optimal heat exchanger network arise from the extremely large number of possible stream combinations. Algorithmic methods, such as branch-and-bound method ${ }^{4)}$, tree search method $^{7)}$, depth first tree search method ${ }^{91}$, and implicit enumeration method ${ }^{2}$, have been developed to reduce the combinatorial problem, but application of these methods requires some special mathematical background and computational skill, and the optimality of the produced network is not guaranteed. Alternative approaches, such as the heuristic method ${ }^{83}$ and systematic methods ${ }^{1,5,6}$, have been developed to avoid the combinatorial problem. The heuristic method, always matching the hot stream of highest

Received August 4, 1981. Correspondence concerning this article should be addressed to $\mathrm{M}$. Muraki. supply temperature with the cold stream of highest target temperature, was developed by Ponton and Donaldson $^{8}$. This method, in many cases, generates surprisingly good networks, but it sometimes generates networks which are quite far from optimal. This is mainly caused by insufficient consideration of maximum heat recovery (maximum amount of heat exchange among hot and cold streams) and optimal locations of heaters and coolers. Rathore and Powers $^{9}$ pointed out that the utility cost associated with heating and cooling is normally more important than the capital cost, so recent works have concentrated on finding networks which achieve maximum heat recovery while showing low capital cost. Systematic methods have been developed by Nishida et $a .^{6)}$, Linnhoff and Flower ${ }^{5)}$, and Flower and Linnhoff ${ }^{11}$, and these methods generate the networks achieving maximum heat recovery. Nishida et al. ${ }^{6}$ ) and Linnhoff and Flower $^{5}$ generated the initial networks, and improved them using evolutionary rules. The former generated the initial networks using the minimum total area algorithm, and the latter generated them using the problem table. The initial networks generated by these methods consist of a large number of units. The calculation load in the evolution stage is in propotion to the number of units in the initial network. It was mentioned that these methods can be implemented by hand calculation without special mathematical background and computational skill, but it is difficult to apply them to large problems because the number of units in the initial network 
increases rapidly as the number of process streams of the problem increases. Flower and Linnhoff ${ }^{1>}$ developed a thermodynamic-combinatorial method using the minimum number of units, but this method could not produce networks for problems having difficult heat recovery situations.

The purpose of this study is to develop a practical method of synthesizing an optimal network by hand calculation, so it is necessary to minimize the total annual cost (utility and capital) of the network and also to reduce the calculation load. The method developed consists of three stages (pre-analysis, generation of initial networks, and evolution). In the pre-analysis stage the thermodynamical restrictions of the synthesis problem are investigated in order to achieve maximum heat recovery, since the utility cost heavily dominates the total cost. The problem table, used in the temperature interval method ${ }^{x_{1}}$, was adopted to determine the minimum utility requirement and also to search the necessary conditions for maximum heat recovery. In the generation stage the initial network under the condition of maximum heat recovery is synthesized by a simple algorithm, intended to decrease the number of units as much as possible and to locate them optimally for reduction of capital cost and calculation load in the evolution stage. In the evolution stage the initial network is improved by evolutionary rules based on streamsplitting, which make possible a reduction in capital cost by increasing the heat duties of units and varying the exchanger sizes. The total annual cost and calculation load in the evolution stage can be greatly reduced by this method. The effectiveness of this method is demonstrated by solving problems which have been reported in previous works.

\section{Formulation of the Problem}

The problem is to synthesize a minimum-cost network of exchangers, heaters, and/or coolers and is stated as follows:

There are $n_{H}$ hot streams with heat capacity flowrates $F_{H i}$ at the inlet temperatures $T_{H 1 i}$ and the target temperatures $T_{H Z 2 i}\left(i=1,2, \cdots, n_{H}\right)$.

There are $n_{C}$ cold streams with heat capacity flowrates $F_{C j}$ at the inlet temperatures $T_{C i j}$ and the tagret temperatures $T_{C 2 j}\left(j=1,2, \cdots, n_{C}\right)$.

Heating and cooling utilities are available at specified conditions, and single-pass heat exchangers of countercurrent type are used. Heat transfer coefficients $U_{i j}$ and heat capacity flowrates $F_{H i}, F_{C j}$ are assumed to be independent of temperature.

The objective function is the total annual cost; which consists of capital and utility costs. The capital cost for the $i^{\text {th }}$ exchanger, heater, and cooler denoted by $C_{E i}, C_{H i}$, and $C_{C i}$, respectively can be

\begin{tabular}{|c|c|c|c|c|c|c|}
\hline & $F_{H}$ & $T_{H 1}$ & $T_{H 2}$ & $F_{C}$ & $T_{C 1}$ & $T_{C 2}$ \\
\hline \multicolumn{7}{|c|}{ Problem 4SP1 } \\
\hline Shl* & $16,666.8$ & 320 & 200 & $\mathrm{Sc} 1 * 14,450.1$ & 140 & 320 \\
\hline Sh2 & $20,000.0$ & 480 & 280 & $\mathrm{Sc} 2 \cdot 11,530.0$ & 320 & 200 \\
\hline \multicolumn{7}{|c|}{ Problem 4SP2 } \\
\hline Sh1 & 20,000 & 500 & 110 & Sc1 70,000 & 25 & 420 \\
\hline Sh2 & 50,000 & 430 & 230 & & & \\
\hline Sh3 & 30,000 & 400 & 110 & & & \\
\hline \multicolumn{7}{|c|}{ Problem 5SP1 } \\
\hline Sh1 & 31,500 & 480 & 250 & Sc1 24,700 & 200 & 400 \\
\hline \multirow[t]{2}{*}{ Sh2 } & 25,200 & 400 & 150 & $\mathrm{Sc} 221,600$ & 100 & 400 \\
\hline & & & & $\mathrm{Sc} 324,500$ & 150 & 360 \\
\hline \multicolumn{7}{|c|}{ Problem 6SP1 } \\
\hline Sh1 & 23,800 & 520 & 300 & Sc1 16,000 & 100 & 430 \\
\hline Sh2 & 28,000 & 440 & 150 & Sc2 26,350 & 200 & 400 \\
\hline Sh3 & 33,600 & 390 & 150 & Sc3 32,760 & 180 & 350 \\
\hline \multicolumn{7}{|c|}{ Problem 7SP1 } \\
\hline Sh1 & 23,800 & 520 & 300 & Sc1 16,000 & 100 & 430 \\
\hline Sh2 & 28,000 & 440 & 150 & Sc2 19,840 & 350 & 410 \\
\hline \multirow[t]{2}{*}{ Sh3 } & 33,600 & 390 & 150 & $\mathrm{Sc} 326,350$ & 200 & 400 \\
\hline & & & & Sc4 32,760 & 180 & 350 \\
\hline \multicolumn{7}{|c|}{ Problem 7SP3 } \\
\hline Sh1 & 153,018 & 675 & 150 & Sc1 478,351 & 60 & 710 \\
\hline Sh2 & 111,220 & 592 & 452 & & & \\
\hline Sh3 & 45,507 & 543 & 115 & & & \\
\hline Sh4 & 598,500 & 428 & 344 & & & \\
\hline Sh5 & 116,482 & 398 & 100 & & & \\
\hline Sh6 1, & $, 277,434$ & 301 & 231 & & & \\
\hline \multicolumn{7}{|c|}{ Problem 8SP1 } \\
\hline Sh1 & 22,400 & 470 & 320 & Sh1 16,800 & 200 & 420 \\
\hline $\mathrm{Sh} 2$ & 17,500 & 450 & 240 & Sh2 23,200 & 150 & 400 \\
\hline $\operatorname{Sh} 3$ & 28,500 & 370 & 150 & Sh3 35,100 & 185 & 330 \\
\hline Sh4 & 20,100 & 310 & 200 & Sh4 17,250 & 140 & 300 \\
\hline \multicolumn{7}{|c|}{ Problem 10SP1 } \\
\hline Sh1 & 23,800 & 520 & 300 & Sc1 11,530 & 240 & 431 \\
\hline $\mathrm{Sh} 2$ & 20,000 & 480 & 280 & $\mathrm{Sc} 2 \quad 16,000$ & 100 & 430 \\
\hline$S h 3$ & 38,000 & .440 & 150 & $\operatorname{Sc} 3 \quad 26,350$ & 200 & 400 \\
\hline Sh4 & 33,600 & 390 & 200 & Sc4 32,760 & 180 & 350 \\
\hline Sh5 & 16,670 & 320 & 200 & Sc5 14,450 & 140 & 320 \\
\hline
\end{tabular}

* Sh (Sc) represents a hot (cold) stream having flow rate $F_{H}\left(F_{C}\right)$, inlet temperature $T_{H 1}\left(T_{C 1}\right)$, and target temperature $T_{H 2}\left(T_{C 2}\right)$.

All capacity heat flowrates are given in $\mathrm{Btu} / \mathrm{hr} \cdot{ }^{\circ} \mathrm{F}$ and temperatures in ${ }^{\circ} \mathrm{F}$.

(continued)

correlated to their corresponding heat transfer areas $A_{E i}, A_{H i}$, and $A_{C i}$ by the empirical expressions $C_{E i}=$ $a A_{E i}^{b}, C_{H i}=a A_{H i}^{b}$, and $C_{C i}=a A_{O i}^{b}$. The total annual cost of the network can be expressed as

$$
J=\delta\left(\sum C_{E i}+\sum C_{H i}+\sum C_{C i}\right)+\sum r_{k} Q_{k} \theta
$$

where $\delta$ is annual rate of terurn, $r$ is unit cost of utility, $Q$ is amount of utility per hour, and $\theta$ is operating hours per year.

The illustrative examples in this paper are all taken from previous works, and their specification of process streams and design data are summarized in Table 1. The minimum allowable approach temperatures are very important design variables, but no attempt has been made to optimize them in this study, as in most previous works, since their optimization should be left to final design with detailed design equations: 


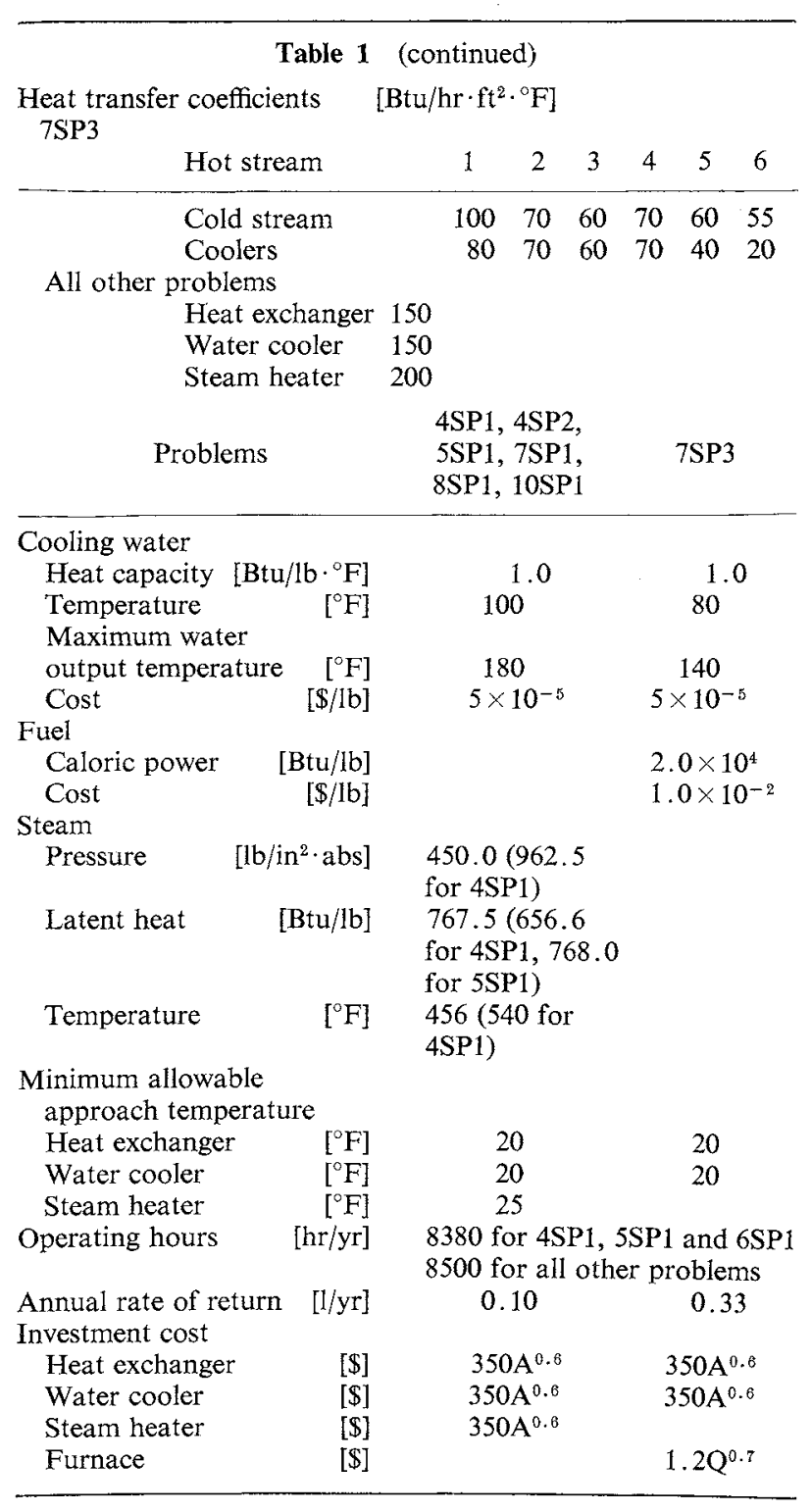

\section{Pre-analysis}

The utility cost associated with heating and cooling is normally more important than the capital cost, so recent work has concentrated on finding networks which achieve maximum heat recovery while showing low capital cost. This study also synthesizes an optimal network under the condition of maximum heat recovery, so it is necessary to calculate minimum utility requirement precisely, and to search the conditions necessary to achieve maximum heat recovery.

The problem table, which was used in the temperature interval method ${ }^{5}$, was adopted in this preanalysis, and the problem table of 7SP3, which is specified in Table 1, is shown in Table 2. Linnhoff and Flower $^{5)}$ described the physical significance of the columns of the problem table. The figures in columns 4 and 5 represent the heat flows into and out of the sub-networks for the case where the minimum
Table 2 Problem table for $7 \mathrm{SP} 3$

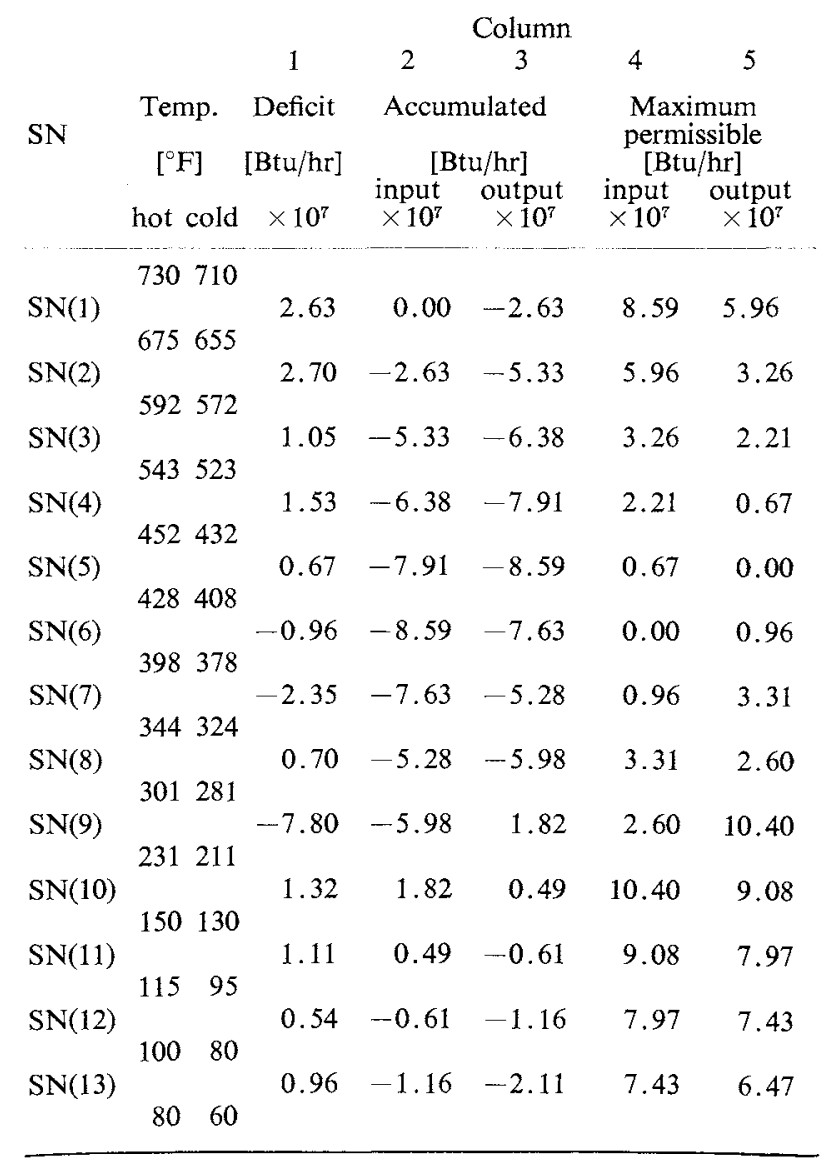

utility heating requirement is received at the highest possible temperature. Thus the top figure in column 4 is the minimum utility heating requirement and the bottom figure in column 5 is the minimum utility cooling requirement. For the case of 7SP3, the minimum utility heating and cooling requirements are $8.58 \times 10^{7}$ and $6.47 \times 10^{7} \mathrm{Btu} / \mathrm{hr}$ respectively. This problem table is further used to search the necessary conditions to achieve maximum heat recovery. For the example of 7SP3, it is clearly necessary to separate 7SP3 into two sub-problems, one consisting of $\mathrm{SN}(1)-\mathrm{SN}(5)$ and the other consisting of $\mathrm{SN}(6)$ $\mathrm{SN}(13)$, because the figure of $\mathrm{SN}(5)$ in column 5 (the figure of $\mathrm{SN}(6)$ in column 4) is 0 . In other words, it indicates that heat flow between these two subproblems does not exist when maximum heat recovery is achieved. If heat is exchanged between these two sub-problems, it is impossible to achieve maximum heat recovery. It is also clear from table 2 that $\mathrm{Scl}$ is necessary to match Sh1 and Sh3 simultaneously in $\mathrm{SN}(5)$, because there exists in $\mathrm{SN}(5)$ a minimum allowable approach temperature constraint produced by decomposition, and it indicates that streamsplitting of $\mathrm{Scl}$ is necessary in $\mathrm{SN}(5)$. This preanalysis is necessary and effective to achieving maximum heat recovery before synthesis of networks. 


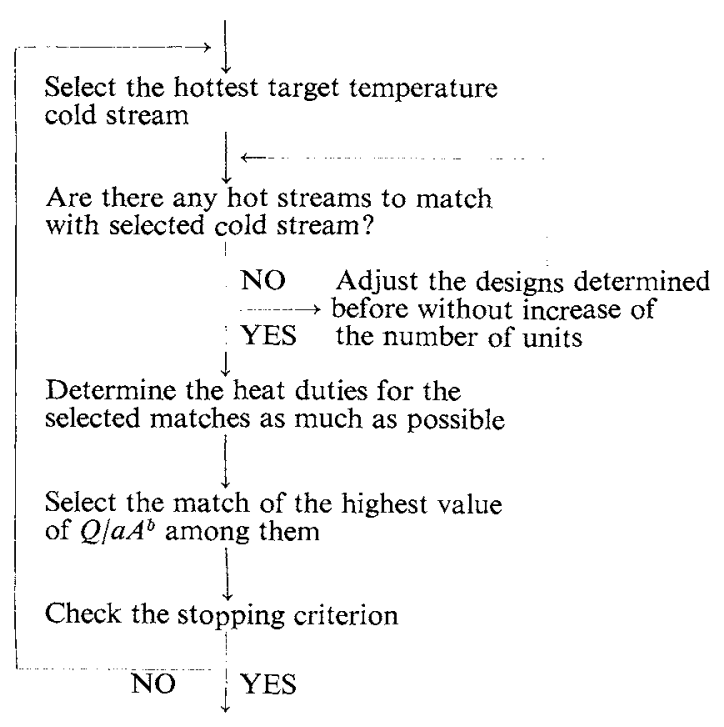

Fig. 1 Algorithm of initial network generation

\section{Generation of Initial Networks}

The purpose of this stage is to generate networks as close to the optimal network as possible, so that the calculation load in the evolution stage can be greatly decreased. Nishida et $a l .^{6}{ }^{\prime}$ generated initial networks which have minimum total heat transfer area. Linnhoff and Flower $^{5}$ generated them from the problem tables mentioned above, and improved them by evolutionary rules. They could generate good networks, but it is necessary for them to calculate the total cost of a large number of neighboring networks, or to consider a large number of possible matches, in the evolution stage before producing the optimal network. The heuristic method ${ }^{8}$, always matching the hot stream of highest supply temperature with the cold stream of highest target temperature, can generate surprisingly good networks in many cases, but sometimes it generates networks which are quite far from optimal. This is due to insufficient consideration of maximum heat recovery and the optimal locations of heaters and coolers in this method.

Considering these results, a simple algorithm is developed to generate nearly optimal networks, which achieve maximum heat recovery while showing low capital costs. Capital costs depend on the combinations of streams and the number of units, and these two factors strongly interact with each other. The algorithm is shown in Fig. 1. The results of the preanalysis stage are adopted to achieve maximum heat recovery, and available utilities are treated as process streams to determine the optimal locations of heaters and coolers. The matching rule of the minimum area algorithm ${ }^{6)}$ results in an increase of calculation load in the evolutionary stage because the network generated consists of many small units, and the
(1) $F_{H}>F_{C}$
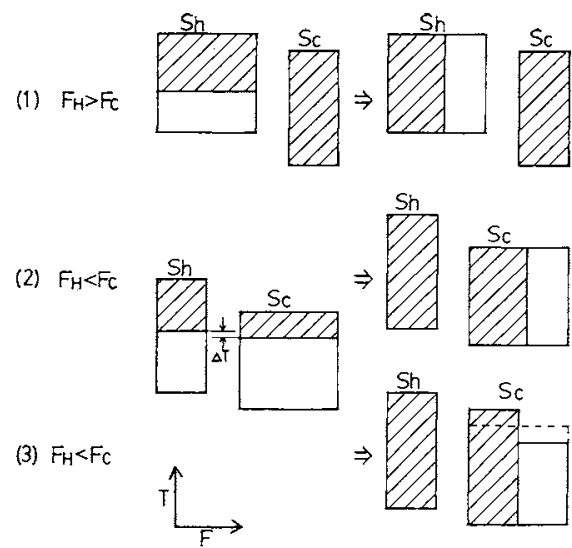

Fig. 2 Evolutionary rules of increasing heat duty

minimum-area network is not the minimum-cost network because the power factor of costing equations for heat transfer area is less than 1.0. So a new matching rule is developed to generate nearly optimal networks. This rule is that the highest target temperature cold stream is matched with the hot stream having the highest value of $Q / a A^{b}$. The initial networks of this study correspond to those which are improved by evolutionary rules ${ }^{5,6}$ because the matches are determined in consideration of cost performance. In the majority of cases this rule generates the same matches that the rule of the hottest hot with the hottest cold streams ${ }^{8)}$ generates. When an infeasible match is encountered during the generation of the initial network, firstly the heat duties of the units are modified in the reverse order of generation without increase of number of units. If this correction is not effective, the matches determined before rearranged in the reverse order of generation so as to solve this infeasible match.

\section{Evolution}

The purpose of this stage is to reduce the capital cost of the network under the condition achieving maximum heat recovery. Simple evolutionary rules are adopted in this study because the network generated in the previous stage is nearly optimal. If the power factor of the costing equation for heat transfer area is less than 1.0, two ways are effective for capital cost reduction. One is to reduce the number of units, and the other is to vary exchanger sizes, as pointed out by Nishida et al. ${ }^{6}$, and Linnhoff and Flower $^{5}$.

It is essential for reduction of the number of units to increase heat duties of the units, and streamsplitting is effective for this. There are three cases where stream-splitting can increase heat duties of units, as shown in Fig. 2.

Stream-splitting is sometimes effective in varying exchanger sizes without influencing the other 
(1)

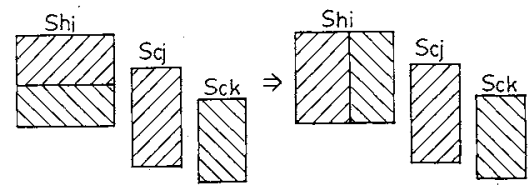

(2)

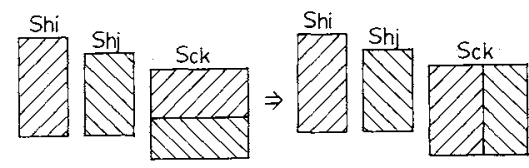

Fi. 3 Evolutionary rules of varying exchanger sizes

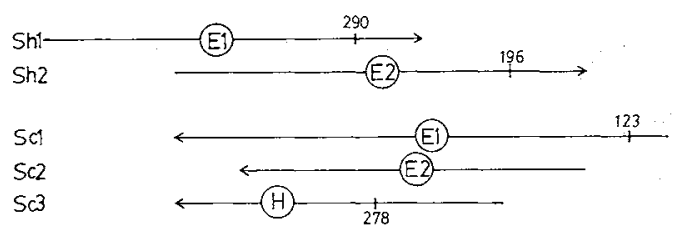

Fig. 4 Progress of initial network generation for 5SP1 problem

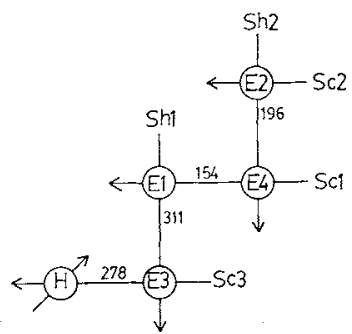

Fig. 5 Initial network for 5SP1 problem

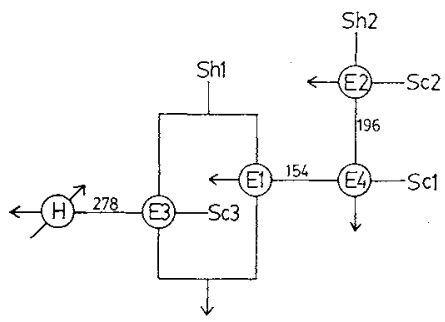

Fig. 6 Final network for 5SP1 problem

matches ${ }^{5}$, but a comparison of costs is necessary to decide whether this evolutionary rule should be adopted or not. Figure 3 shows this rule.

The simple evolutionary rules mentioned above are applied to improve the initial network according to their order of generation. Not only the capital cost but also the calculation load in the evolution stage can be decreased greatly.

\section{Illustrative Example}

This method is applied to the synthesis problems specified in Table 1 to demonstrate its effectiveness. The calculation loads can be decreased greatly in comparison with the systematic methods ${ }^{1, \overline{5}, 6)}$ mentioned above, but the effectiveness in decreasing the
Table 3 Problem table for 5SP1

\begin{tabular}{|c|c|c|c|c|c|c|}
\hline \multirow[t]{2}{*}{ SN } & \multirow{2}{*}{$\begin{array}{l}\text { Temp. } \\
{\left[{ }^{\circ} \mathrm{F}\right]} \\
\text { hot cold }\end{array}$} & \multirow{2}{*}{$\begin{array}{c}\text { Deficit } \\
\text { [Btu/hr] } \\
\times 10^{6}\end{array}$} & \multicolumn{2}{|c|}{$\begin{array}{l}\text { Accumulated } \\
{[\text { Btu/hr] }}\end{array}$} & \multicolumn{2}{|c|}{$\begin{array}{c}\text { Maximum } \\
\text { premissible } \\
\text { [Btu/hr] }\end{array}$} \\
\hline & & & $\begin{array}{l}\text { input } \\
\times 10^{6}\end{array}$ & $\begin{array}{l}\text { output } \\
\times 10^{6}\end{array}$ & $\begin{array}{l}\text { input } \\
\times 10^{8}\end{array}$ & $\begin{array}{l}\text { output } \\
\times 10^{6}\end{array}$ \\
\hline & 480460 & & & & & \\
\hline SN(1) & 420400 & -1.89 & 0.00 & 1.89 & 3.02 & 4.91 \\
\hline $\mathrm{SN}(2)$ & $400 \quad 380$ & 0.30 & 1.89 & 1.59 & 4.91 & 4.61 \\
\hline $\mathrm{SN}(3)$ & 380360 & -0.21 & 1.59 & 1.80 & 4.61 & 4.82 \\
\hline $\mathrm{SN}(4)$ & $250 \quad 230$ & 1.83 & 1.80 & -0.03 & 4.82 & 2.99 \\
\hline $\mathrm{SN}(5)$ & & 1.37 & -0.03 & -1.40 & 2.99 & 1.62 \\
\hline $\mathrm{SN}(6)$ & $170 \quad 150$ & 1.05 & -1.40 & -2.44 & 1.62 & 0.58 \\
\hline $\mathrm{SN}(7)$ & $150 \quad 130$ & -0.07 & -2.44 & -2.37 & 0.58 & 0.65 \\
\hline $\mathrm{SN}(8)$ & 120100 & 0.65 & -2.37 & -3.02 & 0.65 & 0.00 \\
\hline
\end{tabular}

calculation loads cannot be evaluated quantitatively. It is proved that this method is very effective for problems which must be separated into two subproblems, such as $7 \mathrm{SP} 3$ and $8 \mathrm{SP} 1$. Problems 5SP1 and $7 \mathrm{SP} 3$ are solved to illustrate this method.

Problem 5SP1 The problem table representing the 5SP1 problem is shown in Table 3, and it is determined that minimum utility heating requirement is $3.02 \times$ $10^{6} \mathrm{Btu} / \mathrm{hr}$, and that the portion less than $170^{\circ} \mathrm{F}$ of $\mathrm{Sh} 2$ must be matched with Sc1. Both $\mathrm{Sc} 1$ and Sc3 are selected as the cold streams of highest target temperature, and the values of $Q / a A^{b}$ are respectively calculated for feasible matches, Sc1-Sh1, Sc1-Steam, Sc3-Sh1, and Sc3-Steam, Sc1-Sh1 is selected and represented as E1. Sc3-Steam and Sc2-Sh2 are selected and represented as $\mathrm{H}$ and $\mathrm{E} 2$. It is impossible to match the portion less than $277.81^{\circ} \mathrm{F}$ of Sc3 with hot streams, as shown in Fig. 4. According to the algorithm of initial network generation, heat duty of E2 cannot be modified without increasing the number of units, but $\mathrm{E} 1$ is possible, so $\mathrm{E} 1$ is modified to be able to match the portion of less than $277.81^{\circ} \mathrm{F}$ of Sc3. Figure 5 shows the initial network of the 5SP1 problem generated by this method with a total annual cost of $\$ 39,013 / \mathrm{yr}$. Next, the application of evolutionary rules gives an improved network with a total annual cost of $\$ 38,713 / \mathrm{yr}$, as shown in Fig. 6. The same network was generated by Nishida et al. ${ }^{6}$, and Linnhoff and Flower ${ }^{5)}$ further optimized the streamsplitting ratio, but this study leaves this optimization to final design.

Problem 7SP3 This problem corresponds to a crude oil heating train in which a furnace is used instead of a steam heater and the heat transfer coefficients vary with the combinations of streams. The problem 
table is shown in Table 2. As mentioned above, it is necessary to separate this problem into two subproblems, one consisting of $\mathrm{SN}(1)-\mathrm{SN}(5)$ and the other consisting of $\mathrm{SN}(6)-\mathrm{SN}(13)$, and the minimum utility heating and cooling requirements are $8.58 \times 10^{7}$ and $6.47 \times 10^{7} \mathrm{Btu} / \mathrm{hr}$ respectively. The initial networks, generated separately for the two sub-problems, are shown in Fig. 7. Next, evolutionary rules are applied separately and Fig. 8 shows the improved network with a total annual cost of $\$ 1,181,295 / \mathrm{yr}$. Grossmann and Sargent ${ }^{2)}$ generated the networks using the implicit enumeration method, but the total annual cost of their network was $\$ 1,199,158 / \mathrm{yr}$, because maximum heat recovery is not achieved in their network. Table 4 shows the calculation results in comparison with recent work. It is clear that this method can generate networks equal to those generated by other methods or better.

\section{Conclusion}

A practical synthesis method of heat exchanger network, which can be implemented by hand calculation, is developed and applied to problems reported in previous works. This method consists of preanalysis, initial network generation, and evolution stages. In the pre-analysis stage, the problem table is used to determine the minimum utility requirement and necessary conditions. Separation into subproblems is effective in achieving maximum heat recovery. In the initial network generation stage, nearly optimal networks are generated, considering maximum heat recovery and cost performance of units. In the evolution stage, the initial networks are improved by simple evolutionary rules, increasing heat duties of units and varying exchanger sizes.

The calculation loads can be decreased greatly in comparison with systematic methods ${ }^{1,5,6)}$, but the effectiveness in decreasing the calculation loads cannot be evaluated quantitatively. It is clear that this method can generate networks equal to those generated by other methods or better.

\footnotetext{
Nomenclature

A $=$ heat transfer area

$a \quad=$ parameter of cost function

$=$ parameter of cost function

C $\quad$ = capital cost of unit

$F \quad=$ heat capacity flowrate

$J \quad=$ total annual cost

$n \quad=$ number of streams

$Q \quad=$ heat to be transferred

$r \quad=$ unit cost of utility

$T \quad=$ temperature

$U \quad=$ heat transfer coefficient

$\delta \quad=$ annual rate of return
}

$\left[\mathrm{Btu} / \mathrm{hr} \cdot{ }^{\circ} \mathrm{F}\right]$

[\$/y]

[-]

[Btu/hr]

[\$/Btu]

$\left[{ }^{\circ} \mathrm{F}\right]$

$\left[\mathrm{Btu} / \mathrm{hr} \cdot{ }^{\circ} \mathrm{F} \cdot \mathrm{ft}^{2}\right]$

$[1 / y r]$

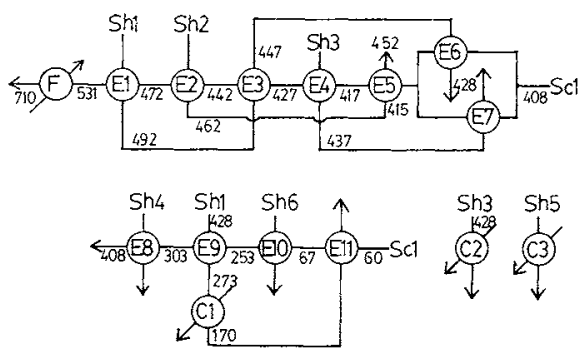

Fig. 7 Initial network for 7SP3 problem

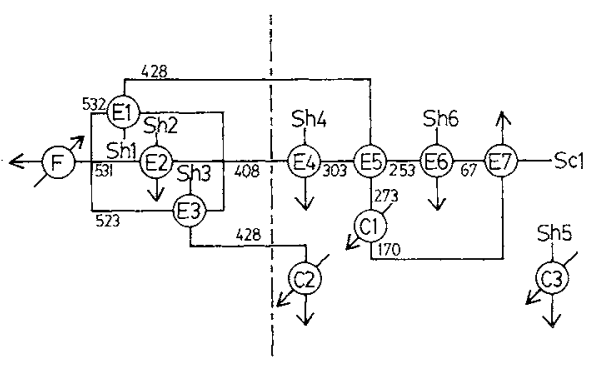

Fig. 8 Final network for 7SP3 problem

\begin{tabular}{|c|c|c|c|}
\hline & Table 4 & $\begin{array}{l}\text { alculation results } \\
\text { [\$/yr] } \\
\text { Nishida et al. }{ }^{6)}\end{array}$ & Grossmann $^{2}$ \\
\hline $4 \mathrm{SP} 1$ & 13,590 & 13,590 & 13,590 \\
\hline $4 \mathrm{SP} 2$ & 19,655 & 20,353 & 21,663 \\
\hline 5SP1 & 38,713 & 38,713 & 38,288 \\
\hline 6SP1 & 35,010 & 35,010 & 35,010 \\
\hline 7SP1 & 30,172 & - & 30,172 \\
\hline 7SP3 & $1,181,295$ & 一 & $1,199,158$ \\
\hline $8 \mathrm{SP} 1$ & 38,303 & - & 41,228 \\
\hline 10SP1 & 43,884 & 43,984 & 44,160 \\
\hline
\end{tabular}

$\theta$

$=$ operating hours per year

$[\mathrm{hr} / \mathrm{yr}]$

$\langle$ Subscripts〉

$$
\begin{array}{ll}
C & =\text { cold stream, cooler } \\
E & =\text { heat exchanger } \\
H & =\text { hot stream, heater } \\
i & =\text { hot stream number } \\
j & =\text { cold stream number } \\
k & =\text { kind of utility } \\
1 & =\text { inlet } \\
2 & =\text { target }
\end{array}
$$

\section{Literature Cited}

1) Flower, J. R, and B. Linnhoff: AIChE J., 26, 1 (1980),

2) Grossmann, I. E. and R. W. Sargent: Comput. Chem. Eng., 2, 1 (1978).

3) Hlavacek, V.: ibid., 2, 67 (1978).

4) Lee, K. F., A. H. Masso and D. F. Rudd: Ind. Eng. Chem., Fundam., 9, 48 (1970).

5) Linnhoff, B, and J. R. Flower: AIChE J., 24, 633 (1978).

6) Nishida, N., Y. A. Liu and L. Lapidus: ibid., 23, 77 (1977).

7) Pho, T. K. and L. Lapidus: ibid., 19, 1182 (1973).

8) Ponton, J. W. and R. A. B. Donaldson: Chem. Eng. Sci., 29, 2375 (1974).

9) Rathore, R. N. S. and G. J. Powers: Ind. Eng. Chem., Process Des. Dev., 14, 175 (1975). 